\section{Pest adaptation}

Sir — The News item about the "Dispute over insect resistance to crops" (Nature 388, $817 ; 1997)$ focuses attention on the critical issue of pest adaptation to crops genetically engineered to express insecticidal proteins from the soil bacterium Bacillus thuringiensis $(B t)$. The article appropriately conveys the message that the knowledge required to design effective, scientifically based resistance management plans is not at present available.

However, the article's emphasis on conflict between academic scientists and industry is somewhat misleading, because it is widely recognized that the urgently needed progress will come only with cooperation, rather than confrontation. I am characterized in the article as "one of UCS's [Union of Concerned Scientists'] scientific advisers".

In fact, like other academic scientists, I have expressed my views in publications available to all concerned parties (see Proc. Natl Acad. Sci. USA 94, 3488-3490; 1997) and have provided, upon request, specific information to industry and to the Environmental Protection Agency, as well as to organizations such as UCS. Like other academic scientists, I have concentrated primarily on generating and disseminating knowledge about pest resistance that is essential for improving management strategies. As we struggle together to create management plans based on the scant data available today, the necessity of committing public and private resources to improving understanding of resistance becomes ever clearer.

\section{Bruce Tabashnik}

Department of Entomology,

University of Arizona,

Tucson, Arizona 85721, USA

e-mail:brucet@ag.arizona.edu

\section{Value for money}

\section{inUS laboratories}

Sir — Frederick Sachs argues that the number of US National Institutes of Health (NIH) grants should be limited to two per investigator in order to fund additional young investigators (Nature 388, 222; 1997). Although there may be merits to limiting further funding to investigators with a large number of grants or resources, the suggested limit would be too restrictive.

Such sums would support only a relatively small laboratory. Clearly the best qualified senior scientists can and should be able to support significantly larger and more productive research laboratories. Unfortunately, Sachs fails to provide proof of his assertion that shifting funds from larger laboratories to a greater number of smaller laboratories would provide more major discoveries.

His discussion on Nobel prizes is irrelevant. Very few scientists will receive such an accolade and often it will be for a body of work spanning their career rather than a single discovery. This type of award system cannot recognize the excellent and critical research performed by thousands of other researchers. Indeed, major discoveries are usually built on a framework of preceding supportive work that comprises most of the funding from NIH and other agencies and which accounts for the majority of scientific literature.

The criterion of performance per existing research dollar by an investigator should be established and used to guide future funding decisions. The research dollars would include all funding available to an investigator and his or her laboratory, including research grants, endowments, awards to graduate and postdoctoral students, industrial support and donations.

Of course, any judgement of academic performance (number and impact factor of publications, for example) does include an arbitrary component, but the suggested formula is more quantifiable than existing 
procedures. For example, a junior investigator with one grant totalling $\$ 100,000$ and one article in Nature has performed 2.5 times better than a senior investigator with grants totalling \$1 million and four papers in this journal, and should thus receive priority for an increase in funding over the senior investigator. The application by granting agencies of this formula for high-risk grants could also be useful in an era when 'safe science' grants often score better than they necessarily should.

\section{Robert D. Nicholls}

Department of Genetics, Case Western Reserve

University School of Medicine and Center for Human Genetics,

University Hospitals of Cleveland,

Cleveland, Ohio 44106, USA

e-mail:rxn19@po.cwru.edu

\section{A probable paradox}

Sir - In September 1993, in your journal, we applied Gott's formulae ${ }^{1}$ to predict that the then Conservative UK government would, with $95 \%$ confidence, continue for a period between 4.3 months and 546 years ${ }^{2}$.

In fact the Conservative government lost office 3.6 years later, so that the prediction could be regarded as confirmed ${ }^{3}$. The success of our prediction naturally pleases us. As we see it, however, there are two problems with this minor success story.

First, had the Conservatives retained power, our prediction would still not be violated. Labour or Conservative victories would both have been in agreement with our inequality. Indeed, any electoral outcome during our lifetimes, or those of our children's children for that matter, would be in agreement with our prediction!

Second, Gott's paper ${ }^{1}$ is now 51 months old. It has not so far been refuted (see ref. 4 for a catalogue of successful predictions). Nevertheless, experience has shown that any theory in physics, however successful, is only an approximation to reality and will eventually be refuted and require modification.

Thus, assuming the Copernican hypothesis with respect to the refutation of Gott's theory, and applying the conclusions of Gott's theory to his theory, then with $95 \%$ confidence it will remain unrefuted for at least 51/39 1.3 months. We can regard this condition as satisfied.

In addition, however, we predict with 95\% confidence that it will have been refuted after $39 \times 51$ months, that is, 165.75 years. Thus we have the paradox that, on the one hand, if the theory is refuted within the next 166 years, then its predictions will be verified. On the other hand, if it is not refuted within the next 166 years then it does not satisfy the prediction that, with $95 \%$ confidence, it should have failed by the year 2163 and is therefore probably wrong, with $95 \%$ confidence. We are here clearly close to a Russell-type paradox, albeit in terms of probabilities.

We cannot use a $100 \%$ confidence limit in the argument, as the upper limit of permitted values then becomes infinitely large and the theory becomes meaningless. On the other hand, use of any upper confidence limit (less than 100\%) makes the theory vulnerable to a Russell-type paradox. It is saved from this paradox only by the existence of this probability.

We conclude that, while the Gott argument can undoubtedly be enlightening in individual cases, as a general theory it is subject to serious shortcomings.

\section{P. T. Landsberg}

J. N. Dewynne

Faculty of Mathematical Studies,

University of Southampton,

Southampton SO17 1BJ, UK

e-mail:ptl@maths.soton.ac.uk

jnd@maths.soton.ac.uk

1. Gott, J. R. Nature 363, 315-319 (1993).

2. Landsberg, P. T., Dewynne, J. N. \& Please, C. P. Nature 365, 384 (1993).

3. Gott, J. R. Nature 387, 842 (1997).

4. Gott, J. R. in Clusters, Lensing and the Future of the Universe (Trimble, V. \& Reisenegger, A., eds) 140 (Astron. Soc. Pacific Conf. Series, Vol 88, 1996). 